\title{
Dados do Inquérito Brasileiro de Nutrição Oncológica em Pediatria: Estudo Multicêntrico e de Base Hospitalar
}

doi: https://doi.org/10.32635/2176-9745.RBC.2021v67n4.1289

\author{
Data from the Brazilian Survey of Pediatric Oncology Nutrition: Multicenter, Hospital-Based Study \\ Datos de Encuesta Brasileña de Nutrición en Oncología Pediátrica: Estudio Multicéntrico Hospitalario
}

Nivaldo Barroso de Pinho'; Wanélia Vieira Afonso ${ }^{2}$; Patricia de Carvalho Padilha ${ }^{3}$; Wilza Arantes Ferreira Peres ${ }^{4}$; Carolina Fernandes de Macedo Soares ${ }^{5}$; Juliana Silva do Nascimento Braga ${ }^{6}$; Arthur Orlando Corrêa Schilithz ${ }^{7}$; Viviane Dias Rodrigues ${ }^{8}$; Renata Brum Martucci ${ }^{9}$

\section{RESUMO}

Introdução: A desnutrição é observada em crianças com câncer e está associada a desfechos clínicos negativos. Objetivo: Descrever a prevalência de inadequação do estado nutricional de crianças e adolescentes com neoplasia maligna na admissão hospitalar em Centros de Referência do câncer infantil no Brasil. Método: Estudo transversal aninhado a um estudo de coorte, multicêntrico, de base hospitalar. A amostra probabilística foi feita em dois estágios em cada estrato por Macrorregião pelo método de probabilidade proporcional ao tamanho com um ano de coleta em cada instituição. Foram coletados em 13 instituiçóes de referência dados clínicos, antropométricos, de composição corporal e sobre o questionário de Avaliação Nutricional Subjetiva Global Pediátrica (ANSGP), em até 48 horas da admissão hospitalar, entre março de 2018 e agosto de 2019. Resultados: O estudo totalizou 723 pacientes nas cinco regiōes do Brasil. A prevalência de desnutrição moderada e grave foi de $25,9 \%$ na faixa etária de 2 a 5 anos, 40,1\% de 5 a 10 anos e $39,7 \%$ de 10 a 19 anos, de acordo com ANSGP. Segundo o Índice de Massa Corporal/Idade (IMC/I), magreza e magreza acentuada totalizaram 13\%, risco de sobrepeso, sobrepeso e obesidade apresentaram uma prevalência de $26,7 \%$ de 2 a 5 anos; $24,9 \%$ de 5 a 10 anos; e $25,7 \%$ de 10 a 19 anos. Conclusáo: Evidenciou-se alta prevalência de inadequação nutricional pela ANSGP, sugerindo que a desnutrição pode ser subdiagnosticada quando utilizado somente o IMC/I, fortalecendo a necessidade de utilizaçáo de métodos complementares na avaliaçáo nutricional de crianças com câncer.

Palavras-chave: Estado Nutricional; Pediatria; Neoplasias; Inquéritos Epidemiológicos.

\section{ABSTRACT}

Introduction: Malnutrition is found in children with cancer and is associated with negative clinical outcomes. Objective: To describe the prevalence of inadequate nutritional status of children and adolescents with malignant neoplasm at hospital admission in childhood cancer reference centers in Brazil. Method: Cross-sectional study nested in a multicenter, hospital-based cohort study. The probabilistic sample was carried out in two stages in each stratum by macro-region using the probability method proportional to the size with one year of collection in each institution. Clinical, anthropometric, body composition data and the Pediatric Subjective Global Nutritional Assessment (SGNA) questionnaire were collected from 13 reference institutions within 48 hours of hospital admission, from March 2018 to August 2019. Results: The study totaled 723 patients in the 5 regions of Brazil. The prevalence of moderate and severe malnutrition was $25.9 \%$ in the age group of 2 to 5 years, $40.1 \%$ in 5 to 10 years and $39.7 \%$ in 10 to 19 years, according to the SGNA. According to the Body Mass/Age Index (BMI/I), thinness and marked thinness totaled 13\%, risk of overweight, overweight and obesity showed a prevalence of $26.7 \%$ from 2 to 5 years, $24.9 \%$ from 5 to 10 years and $25.7 \%$ from 10 to 19 years. Conclusion: There was a high prevalence of nutritional inadequacy by the SGNA, suggesting that malnutrition can be underdiagnosed when using only the BMI/I, strengthening the need to use complementary methods in the nutritional assessment of children with cancer.

Key words: Nutritional Status; Pediatrics; Neoplasms; Health Surveys.

\section{RESUMEN}

Introducción: La desnutrición se observa en niños con cáncer y se asocia con resultados clínicos negativos. Objetivo: Describir la prevalencia del estado nutricional inadecuado de niños y adolescentes con neoplasia maligna al ingreso hospitalario en centros de referencia de cáncer infantil en Brasil. Método: Estudio transversal anidado en un estudio de cohorte hospitalario multicéntrico. La muestra probabilística se realizó en dos etapas en cada estrato por macrorregión utilizando el método de probabilidad proporcional al tamaño con un año de recolección en cada institución. Se recopilaron datos clínicos, antropométricos, de composición corporal y el cuestionario Global Pediatric Subjective Nutritional Assessment (ANSGP) de 13 instituciones de referencia dentro de las 48 horas posteriores al ingreso hospitalario, desde marzo de 2018 hasta agosto de 2019. Resultados: El estudio totalizó 723 pacientes en las 5 regiones de Brasil. La prevalencia de desnutrición moderada y severa fue de $25,9 \%$ en el grupo de edad de 2 a 5 años, $40,1 \%$ de 5 a 10 años y 39,7\% de 10 a 19 años, según la ANSGP. Según el Índice de Masa Corporal/ Edad (IMC/I), la delgadez y la delgadez marcada totalizaron $13 \%$, el riesgo de sobrepeso, sobrepeso y obesidad mostró una prevalencia de $26,7 \%$ de 2 a 5 años, $24,9 \%$ de 5 a 10 ańos y 25,7\% de 10 a 19 ańos. Conclusión: Hubo una alta prevalencia de insuficiencia nutricional por parte de la ANSGP, lo que sugiere que la desnutrición puede ser infradiagnosticada cuando se utiliza solo el IMC/I, fortaleciendo la necesidad de utilizar métodos complementarios en la evaluación nutricional de los nińos con cáncer.

Palabra clave: Estado Nutricional; Pediatría; Neoplasias; Encuestas Epidemiológicas.

'Sociedade Brasileira em Nutrição Oncológica (SBNO). Rio de Janeiro (RJ), Brasil. E-mail: suporte@sbno.com.br. Orcid iD: https://orcid.org/0000-0002-1438-168X 2Universidade Federal do Rio de Janeiro (UFRJ), Instituto de Nutrição Josué de Castro (INJC). Instituto Nacional de Câncer José Alencar Gomes da Silva (INCA), Hospital do Câncer I (HCl), Seção de Nutrição e Dietética. Rio de Janeiro (RJ), Brasil. E-mail: nutri.wanelia@uol.com.br. Orcid iD: https://orcid.org/0000-0002-5315-5730 ${ }^{3}$ UFRJ/INJC. UFRJ/Instituto de Puericultura e Pediatria Martagão Gesteira (IPPMG). Bolsista de Produtividade do Conselho Nacional de Pesquisa (CNPq). Rio de Janeiro (RJ), Brasil. E-mail: patricia@nutricao.ufrj.br. Orcid iD: https://orcid.org/0000-0003-0221-7732

${ }^{4}$ UFRJ/INJC. Bolsista de Produtividade do CNPq. Rio de Janeiro (RJ), Brasil. E-mail: wilza@nutricao.ufrj.br. Orcid iD: https://orcid.org/0000-0003-0269-5363

5,6UFRJ/INJC. Rio de Janeiro (RJ), Brasil. E-mails: macedosoares.carolina@gmail; julianasnb@gmail.com. Orcid iD: https://orcid.org/0000-0003-3084-7889; Orcid iD: https://orcid.org/0000-0003-3118-874X

${ }^{7}$ INCA. Rio de Janeiro (RJ), Brasil. E-mail: arthur.br@live.com. Orcid iD: https://orcid.org/0000-0003-2457-3965

${ }^{8}$ INCA/HCI/Seção de Nutrição e Dietética. SBNO. Rio de Janeiro (RJ), Brasil. E-mail: viviane.rodrigues@inca.gov.br. Orcid iD: https://orcid.org/0000-0003-2243-438X ${ }^{9}$ INCA/HCI/Seção de Nutrição e Dietética. SBNO. Instituto de Nutrição da Universidade do Estado do Rio de Janeiro. Rio de Janeiro (RJ), Brasil. E-mail: renata.martucci@inca.gov.br. Orcid iD: https://orcid.org/0000-0002-3354-4229

Endereço para correspondência: INCA/HCI/Seção de Nutrição e Dietética. Praça Cruz Vermelha, 23, 5 andar - Centro. Rio de Janeiro (RJ), Brasil. CEP $20230-130$. 


\section{INTRODUÇÃO}

A estimativa mundial de incidência do câncer na criança de 0 a 14 anos encontra-se em torno de 400 mil casos anuais ${ }^{1}$. A maioria dos casos diagnosticados é de países de baixa e média rendas e, segundo a Organização das Naçôes Unidas, um em cada três desses países apresentam índices extremos de má nutriçãao ${ }^{2,3}$. No Brasil, um total de 8.460 casos novos de câncer pediátrico na faixa etária de 1 a 19 anos foram estimados para $2020^{4}$. O câncer nessa faixa etária representa a principal causa de morte por doença e é visto como um grave problema de saúde pública ${ }^{4}$.

A literatura é consensual ao reconhecer o impacto da doença oncológica no estado nutricional ${ }^{2}$. No Brasil, dados de crianças e adolescentes saudáveis demonstram que a prevalência de desnutrição e o excesso de peso encontram-se em torno de 6,8\% e 25,8\% respectivamente, sendo este último de maior prevalência nos adolescentes ${ }^{5,6}$. A redução na desigualdade econômica no país, no período de 1996 a 2007, levou à mudança no panorama da nutrição infantil com importante aumento na prevalência da obesidade nas últimas décadas ${ }^{5,7,8}$.

As consequências da desnutrição, assim como do sobrepeso e obesidade no câncer infantojuvenil, em qualquer momento do tratamento, são bem relatadas e associadas ao aumento da morbidade e mortalidade. Em contrapartida, a adequação do estado nutricional ao longo da terapia tem sido relacionada com a melhor resposta ao tratamento, menor toxicidade e aumento da sobrevida desses pacientes ${ }^{9,10}$.

Nesses pacientes, a prevalência de má nutrição, seja a desnutrição ou excesso de peso, varia de $8 \%$ a $60 \%$ conforme o tipo de neoplasia, estadiamento da doença e modalidade de tratamento ${ }^{11}$. Em revisão sistemática recente, poucos estudos foram encontrados sobre estado nutricional em crianças com câncer no Brasil. Estudos com dados mais consistentes publicados são em grande parte da Regiáo Sudeste ${ }^{12}$ e não mostram uma representatividade nacional.

A avaliação nutricional na criança com câncer é sem dúvida um grande obstáculo na prática clínica, visto que métodos antropométricos, que dependem do peso corporal, comumente utilizados em crianças saudáveis, por vezes, apresentam limitaçóes no paciente pediátrico com câncer, principalmente naqueles com massa tumoral volumosa, tornando-os inadequados ${ }^{13}$.

Dada a inexistência de estudos multicêntricos sobre estado nutricional em crianças com câncer no Brasil e a fim de dar continuidade às pesquisas do inquérito brasileiro de nutrição oncológica ${ }^{14,15}$, com dados prévios de adultos e idosos, foi realizado o Inquérito Brasileiro de Nutrição Oncológica em Pediatria $(\text { IBNOPe })^{16}$ cujo objetivo é, nessa primeira publicação, descrever a prevalência de inadequação do estado nutricional de crianças e adolescentes com neoplasia maligna na admissão hospitalar em Centros de Referência para o tratamento do câncer infantil no Brasil.

\section{MÉTODO}

Trata-se de um estudo transversal aninhado a um estudo de coorte, multicêntrico e de base hospitalar, conduzido em parceria com a Sociedade Brasileira de Nutrição Oncológica (SBNO), o Serviço de Nutrição do Hospital do Câncer I (HCI) do Instituto Nacional de Câncer José Alencar Gomes da Silva (INCA), o Instituto de Nutrição Josué de Castro da Universidade Federal do Rio de Janeiro e os Centros de Referência para tratamento do câncer infantil nas diferentes Regióes do Brasil, que reuniram informaçóes de crianças e adolescentes no momento da admissáo em Centros de Referência para o tratamento do câncer de todas as Regiōes do Brasil, no período de março de 2018 a agosto de 2019.

Foram considerados elegíveis pacientes de ambos os sexos, na faixa etária de 2 a 19 anos, com diagnóstico de neoplasia maligna confirmada e em vigência de tratamento. Pacientes em cuidados paliativos, em fim de vida, admitidos em Centro de Tratamento Intensivo Pediátrico, com síndrome genética, má formaçôes e portadores do vírus HIV foram excluídos da pesquisa, assim como aqueles cujos responsáveis ou pacientes não concordaram com a participação no estudo.

A ferramenta subjetiva escolhida para coleta de dados avalia indivíduos com idade entre 2 e 19 anos, o que determinou a escolha da faixa etária a ser estudada ${ }^{17,18}$. A pesquisa foi submetida e aprovada pelo Comitê de Ética em Pesquisa do INCA, como instituição proponente (CAAE: 72541617.8.1001.5274) e em todos os Centros participantes. Todos os responsáveis assinaram o Termo de Consentimento Livre e Esclarecido (TCLE) e as crianças acima de 12 anos, o Termo de Assentimento Livre e Esclarecido (TALE).

Para descrição dos dados, foram calculadas as medidas de tendência central e de dispersão para as variáveis quantitativas e tabelas de frequência e de contingência para verificar as características de uma variável em função de outras características. Foram elaborados gráficos de barras para as variáveis qualitativas. Todos os dados foram processados pelo software SPSS versão 26.

A seleção da amostra foi realizada considerando que a proporção de estado nutricional em crianças com diagnóstico de câncer pode variar de acordo com a Regiáo geográfica. Assim, para o cálculo amostral, foram levados 
em consideração os estratos por Macrorregião do país (Regiōes Norte, Nordeste, Sudeste, Sul e Centro-Oeste). Nesse contexto, foram listados os hospitais que prestavam atendimento oncológico pediátrico de acordo com o Sistema Nacional de Informaçóes sobre Estabelecimentos de Saúde. Uma amostra probabilística em dois estágios foi elegível em cada estrato; no primeiro, selecionaram-se os hospitais, utilizando a metodologia de Probabilidade Proporcional ao Tamanho (PPT), e depois as crianças nos hospitais no momento da internação hospitalar.

O tamanho da amostra tem poder de $80 \%$ para detectar diferenças de estado nutricional entre tumores sólidos e hematológicos na ordem de 59\% a 79\% (diferenças de pelo menos 20\%) entre grandes Regiôes geográficas, exceto pela Região Norte.

O número de crianças amostradas inicialmente foi de 1.380, compondo um total de 15 hospitais amostrados para o estudo, os quais equivalem a $34,9 \%$ de todos os hospitais com 300 ou mais internaçóes totais em 2016. O número de hospitalizaçóes amostradas para cada estrato obedeceu à alocação proporcional, como no mínimo 119 crianças (número necessário para comparação entre as Regiōes). No cálculo, esse mínimo não foi atingido na Região Norte em razão do menor número de internaçóes naturalmente existente, compondo apenas a estimativa do estado nutricional para o Brasil. A coleta ocorreu no período de março de 2018 a agosto de 2019.

As instituiçóes selecionadas receberam uma carta-convite direcionada ao Serviço de Nutrição e Dietética para manifestação de interesse com anuência da direçáo-geral da instituição. Após selecionadas, duas instituiçôes recusaram o convite, (1 da Região Sudeste e 1 da Região Nordeste), e foram substituídas automaticamente da lista referente à cada Região, respeitando os mesmos critérios. Depois de cinco meses de início da pesquisa, duas instituiçôes foram desligadas por falta de adesão ao estudo e, a fim de evitar atrasos, a pesquisa seguiu com um total de 13 hospitais participantes, resultando em um número total de 723 crianças.

Após a finalização da coleta, foi realizado um processo de calibração e expansão da amostra, no qual levou-se em consideraçáo todos os cálculos iniciais, bem como o número final de primeiras internaçôes, com resultado equivalente a cerca de 3.600 crianças que receberam atendimento oncológico em todo o país.

A avaliação nutricional objetiva contemplou as medidas antropométricas e de composição corporal e a avaliação nutricional subjetiva foi realizada por meio do exame físico e aplicação do questionário, em até 48 horas da admissão hospitalar. Também foram coletadas informaçóes dos prontuários físicos e eletrônicos dos pacientes, por meio de ficha de coleta de dados elaborada exclusivamente para esta pesquisa. Para caracterização dos dados demográficos e clínicos, foram utilizadas informações do prontuário.

As medidas antropométricas de avaliação do estado nutricional foram: peso, estatura, medidas de composiçáo corporal, como circunferência do braço (CB), dobra cutânea tricipital (DCT), dobra cutânea subescapular (DCS) e circunferência muscular do braço (CMB). Todas as medidas seguiram os padrôes para Avaliação Nutricional de Crianças e Adolescentes, sugeridos pelo Ministério da Saúde ${ }^{19}$ e pela World Health Organization (WHO) ${ }^{20}$. A classificação do estado nutricional foi realizada por meio do escore $z$; e os índices peso/idade, estatura/idade e $\mathrm{IMC} / \mathrm{I}$, classificados segundo a recomendação da $\mathrm{WHO}^{20}$, utilizando o software Anthro e AnthroPlus, sendo o Anthro para criança até 5 anos de idade e AnthroPlus para crianças maiores de 5 anos e adolescentes, na versão 3.2.2 $2^{21}$. As medidas da CB, DCT, DCS e CMB foram classificadas de acordo com a tabela de percentis proposta por Frisancho ${ }^{22}$, na qual utilizaram-se os seguintes pontos de corte: para $\mathrm{CB}$, DCT e DCS $(\mathrm{P}<5=$ desnutrição; $\mathrm{P} \geq 5$ e $\mathrm{P} \leq 95=$ eutrófico; $\mathrm{P}>95$ = obesidade) e $\mathrm{CMB}(\mathrm{P}<5=$ desnutrição; $\mathrm{P} \geq 5$ = eutrófico).

Para pacientes amputados, foram utilizadas as recomendaçôes para paciente pediátrico com câncer do Consenso Nacional de Nutrição Oncológica 2016, que utiliza equação proposta por Osterkamp ${ }^{23}$ no ajuste dos cálculos da avaliação nutricional.

A avaliação nutricional subjetiva foi realizada por meio da aplicação do questionário de Avaliação Nutricional Subjetiva Global Pediátrica (ANSGP) para crianças com câncer e validação de conteúdo por Saraiva et al. ${ }^{17,18}$ nas primeiras 48 horas de admissão hospitalar. Na primeira parte desse questionário, constavam questóes referente à avaliaçáo sobre a história clínica com foco nutricional mediante informações da adequação da estatura e do peso atual, alterações não intencionais no peso, adequação da ingestão dietética, sintomas gastrintestinais, capacidade funcional, estresse metabólico e exame físico (perda de gordura subcutânea, perda muscular e edema). A segunda parte do questionário consistia em informaçóes referentes à anamnese alimentar como o consumo e a frequência da ingestão de alimentos e informaçôes sobre atividades físicas e funcionais. $\mathrm{O}$ estado nutricional foi classificado como normal ou bem nutrido, moderadamente desnutrido e gravemente desnutrido, de acordo com orientação preconizada pelo instrumento de ANSGP ${ }^{17,18}$.

Todas as instituiçóes participantes enviaram um representante, nutricionista do Serviço de Pediatria local, o qual foi denominado supervisor da pesquisa dentro da instituição representada. Esses profissionais participaram do treinamento teórico e prático que antecedeu ao início 
da pesquisa e foi realizado no INCA pelo Serviço de Nutrição do HCI.

Os supervisores de campo de cada unidade foram multiplicadores do treinamento e responsáveis pela conferência nos preenchimentos dos formulários de pesquisa, além de manterem comunicação com a equipe de coordenação. Todos garantiram a existência dos equipamentos necessários para realização da avaliação nutricional objetiva em suas unidades hospitalares (balança com variaçáo de $0,1 \mathrm{Kg}$, estadiômetro com variação de $0,1 \mathrm{~cm}$ e adipômetro Lange ${ }^{\oplus}$ ).

\section{RESULTADOS}

Participaram desta pesquisa 13 Centros de Referência para tratamento do câncer infantil no Brasil (Quadro 1) com um total de 723 crianças, sendo a Região Nordeste a mais prevalente e a Região Norte a menos prevalente na amostra. Destes, $59,8 \%$ eram do sexo masculino e $43,2 \%$ adolescentes de 10 a 19 anos. Nesta casuística, 61,8\% eram portadores de tumores hematológicos. As leucemias foram as neoplasias hematológicas mais prevalentes com $47,9 \%$, seguidas dos linfomas e neoplasias reticuloendoteliais com $14 \%$. Os tumores ósseos com 10,9\% foram o tumor sólido mais prevalente, enquanto o tumor de Sistema Nervoso Central apresentou uma prevalência de 5,0\% (Tabela 1).
A quimioterapia exclusiva foi o tratamento oncológico em vigência mais prevalente $(86,6 \%)$ e a média de tempo de diagnóstico da doença foi de 255 dias.

De acordo com a ANSGP (Tabela 2), 63,8\% foram classificados como normal ou bem nutrido. Entretanto, um quantitativo expressivo de desnutrição moderada $(29,7 \%)$ e grave $(6,5 \%)$ foi identificado segundo o referido método.

Embora mais de $50 \%$ dos pacientes em todas as Regiōes estivessem bem nutridos, as Regiōes Nordeste $(34,6 \%)$ e Centro-Oeste (40\%) apresentaram maior prevalência de pacientes com desnutrição moderada, enquanto os gravemente desnutridos eram da Regiáo Sudeste $(9,4 \%)$. Entre todas, a Regiáo Norte apresentou o maior percentual de pacientes normal ou bem nutridos $(83,3 \%)$ segundo a ANSGP para crianças com câncer.

A Tabela 3 apresenta dados subjetivos e objetivos da avaliação nutricional. Observa-se que, segundo a ANSGP, a prevalência de desnutrição entre aqueles classificados como moderadamente desnutrido e gravemente desnutrido por faixa etária é de $25,9 \%$ de 2 a 5 anos; $40,1 \%$ de 5 a 10 anos; e $39,7 \%$ de 10 a 19 anos.

Segundo o IMC/I, a inadequação do estado nutricional ocorre com maior prevalência para o risco de sobrepeso, sobrepeso e obesidade do que a desnutrição em todas as faixas etárias. Entre as crianças de 2 a 5 anos, 6,4\% apresentam magreza ou magreza acentuada e $26,7 \%$

Quadro 1. Relação dos Centros de Referência para tratamento do câncer na criança da amostra do IBNOPe. Brasil

\begin{tabular}{|c|}
\hline Instituições Participantes do IBNOPe por Região \\
\hline Região Norte \\
\hline Hospital Oncológico Infantil Octávio Lobo - PA \\
\hline Região Nordeste \\
\hline Hospital Oswaldo Cruz - PE \\
\hline Hospital da Liga Norte Riograndense contra o Câncer - RN \\
\hline Hospital Santa Casa de Misericórdia de Maceió - AL \\
\hline Hospital Universitário Lauro Wanderley - Fundação Napoleão Laureano - PB \\
\hline Região Centro-Oeste \\
\hline Hospital de Base do Distrito Federal da Secretaria de Saúde do Distrito Federal - DF \\
\hline Região Sudeste \\
\hline Instituto Nacional de Câncer José Alencar Gomes da Silva - Hospital do Câncer I - RJ \\
\hline Hospital Infantil Darcy Vargas da Secretaria de Estado da Saúde de São Paulo - SP \\
\hline Hospital Infantil Dr. Domingos A. Boldrini. Campinas - SP \\
\hline Hospital Santa Casa de Misericórdia de Belo Horizonte - MG. \\
\hline Região Sul \\
\hline Hospital de Clínicas de Porto Alegre - RS \\
\hline Hospital Infantil Joana de Gusmão - SC \\
\hline Hospital da Criança Santo Antônio - Santa Casa de Misericórdia de Porto Alegre - RS \\
\hline
\end{tabular}

Fonte: Afonso $^{16}$. 
Tabela 1. Características sociodemográficas da amostra do IBNOPe. Brasil $(n=723)$

\begin{tabular}{|c|c|c|c|}
\hline Variáveis & & $\mathbf{n}$ & $\%$ \\
\hline \multicolumn{4}{|l|}{ Região } \\
\hline & Norte & 18 & 2,5 \\
\hline & Nordeste & 269 & 37,2 \\
\hline & Centro-Oeste & 30 & 4,1 \\
\hline & Sudeste & 255 & 35,3 \\
\hline & Sul & 151 & 20,9 \\
\hline \multicolumn{4}{|l|}{ Sexo } \\
\hline & Feminino & 291 & 40,2 \\
\hline & Masculino & 432 & 59,8 \\
\hline \multicolumn{4}{|c|}{ Faixa etária } \\
\hline & De 2 a 5 anos & 189 & 26,1 \\
\hline & De 5 a 10 anos & 222 & 30,7 \\
\hline & De 10 a 19 anos & 312 & 43,2 \\
\hline \multicolumn{4}{|c|}{ Grupo de doenças } \\
\hline & Leucemias & 346 & 47,9 \\
\hline & Linfomas e neoplasias reticuloendoteliais & 101 & 14,0 \\
\hline & $\begin{array}{l}\text { Neoplasias do SNC e neoplasias intracranianas e } \\
\text { intraespinhais mistas }\end{array}$ & 36 & 5,0 \\
\hline & Tumores do sistema nervoso simpático & 39 & 5,4 \\
\hline & Retinoblastoma & 13 & 1,8 \\
\hline & Tumores renais & 39 & 5,4 \\
\hline & Tumores hepáticos & 7 & 1,0 \\
\hline & Tumores ósseos malignos & 79 & 10,9 \\
\hline & Sarcomas de partes moles & 24 & 3,3 \\
\hline & Neoplasias de células germinativas, trofoblásticas, gonadais & 13 & 1,8 \\
\hline & Carcinomas e outras neoplasias epiteliais malignas & 10 & 1,4 \\
\hline & Outras neoplasias malignas inespecíficas & 16 & 2,2 \\
\hline
\end{tabular}

Fonte: Afonso $^{16}$.

Legenda: $\mathrm{SNC}=$ Sistema Nervoso Central.

algum excesso de peso (risco de sobrepeso, sobrepeso ou obesidade). Daquelas entre 5 e 10 anos, $10,8 \%$ com magreza ou magreza acentuada e $24,9 \%$ excesso de peso. O mesmo ocorre entre os adolescentes, $12,8 \%$ apresentam magreza ou magreza acentuada e $25,7 \%$ excesso de peso. Nota-se que com o IMC/I há maior prevalência de excesso de peso nesta amostra. Por outro lado, ao se observarem os resultados da avaliação nutricional subjetiva, percebe-se uma prevalência de desnutrição maior nesta mesma amostra em relação ao IMC/I.

Neste estudo, mais de 90\% ( $\mathrm{n}=720)$ da amostra em todas as faixas etárias apresentaram estatura adequada para idade. Muito baixa estatura foi observada na faixa etária de 2 a 5 anos com $4,3 \%$.
Quanto às medidas de composição corporal, conforme constam na Tabela 3, observa-se, pela CB, que a maior prevalência de desnutrição se encontra na faixa etária de 10 a 19 anos $(23,8 \%)$. O comprometimento de tecido muscular pode ser observado na classificação da CBM, em que $22,7 \%$ na faixa etária de 10 a 19 anos apresenta $\mathrm{P}<5$. O comprometimento de tecido adiposo pela DCT com $\mathrm{P}<5$ foi observado com maior prevalência nas crianças com idade entre 2 e 5 anos de 13,9\%, enquanto a DCS com $\mathrm{P}<5$ foi mais prevalente nos adolescentes entre $10 \mathrm{e}$ 19 anos com 7,7\%.

A Figura 1 apresenta os resultados da classificação do estado nutricional segundo as medidas de composição corporal conforme o sexo e a faixa etária. 
Tabela 2. Avaliação Nutricional Subjetiva Global Pediátrica no momento da internação segundo características da amostra IBNOPe. Brasil $(n=723)$

\begin{tabular}{|c|c|c|c|c|c|c|c|}
\hline & & \multicolumn{6}{|c|}{ ANSGP } \\
\hline & & \multicolumn{2}{|c|}{ Normal/Bem nutrido } & \multicolumn{2}{|c|}{$\begin{array}{l}\text { Moderadamente } \\
\text { desnutrido }\end{array}$} & \multicolumn{2}{|c|}{$\begin{array}{l}\text { Gravemente } \\
\text { desnutrido }\end{array}$} \\
\hline & & $\mathbf{n}$ & $\%$ & $\mathbf{n}$ & $\%$ & $\mathbf{n}$ & $\%$ \\
\hline \multicolumn{8}{|l|}{ Sexo } \\
\hline & Feminino & 196 & 67,4 & 78 & 26,8 & 17 & 5,8 \\
\hline & Masculino & 265 & 61,3 & 137 & 31,7 & 30 & 6,9 \\
\hline \multicolumn{8}{|c|}{ Faixa etária } \\
\hline & De 2 a 5 anos & 140 & 74,1 & 42 & 22,2 & 7 & 3,7 \\
\hline & De 5 a 10 anos & 133 & 59,9 & 80 & 36,0 & 9 & 4,1 \\
\hline & De 10 a 19 anos & 188 & 60,3 & 93 & 29,8 & 31 & 9,9 \\
\hline \multicolumn{8}{|c|}{ Região } \\
\hline & Norte & 15 & 83,3 & 2 & 11,1 & 1 & 5,6 \\
\hline & Nordeste & 161 & 59,8 & 93 & 34,6 & 15 & 5,6 \\
\hline & Centro-Oeste & 16 & 53,3 & 12 & 40,0 & 2 & 6,7 \\
\hline & Sudeste & 156 & 61,2 & 75 & 29,4 & 24 & 9,4 \\
\hline & Sul & 113 & 74,8 & 33 & 21,9 & 5 & 3,3 \\
\hline Brasil & & 461 & 63,8 & 215 & 29,7 & 47 & 6,5 \\
\hline
\end{tabular}

Fonte: Afonso ${ }^{16}$.

Legenda: ANSGP = Avaliaçăo Nutricional Subjetiva Global Pediátrica.

Tabela 3. Estado nutricional segundo avaliação nutricional subjetiva e objetiva de acordo com a faixa etária da amostra do IBNOPe. Brasil $(n=723)$

\begin{tabular}{|c|c|c|c|c|c|c|}
\hline & \multicolumn{6}{|c|}{ Faixa etária } \\
\hline & \multicolumn{2}{|c|}{ De 2 a 5 anos } & \multicolumn{2}{|c|}{ De 5 a 10 anos } & \multicolumn{2}{|c|}{ De 10 a 19 anos } \\
\hline & $\mathbf{n}$ & $\%$ & $\mathbf{n}$ & $\%$ & $\mathbf{n}$ & $\%$ \\
\hline \multicolumn{7}{|l|}{ Classificação ANSGP $(n=723)$} \\
\hline Normal/Bem nutrido & 140 & 74,1 & 133 & 59,9 & 188 & 60,3 \\
\hline Moderadamente desnutrido & 42 & 22,2 & 80 & 36,0 & 93 & 29,8 \\
\hline Gravemente desnutrido & 7 & 3,7 & 9 & 4,1 & 31 & 9,9 \\
\hline \multicolumn{7}{|l|}{ Circunferência do braço $(n=718)$} \\
\hline Desnutrição $(\mathrm{P}<5)$ & 37 & 19,7 & 46 & 21,0 & 74 & 23,8 \\
\hline Eutrófico $(P \geq 5-P \leq 95)$ & 144 & 76,6 & 163 & 74,4 & 220 & 70,7 \\
\hline Obesidade $(P>95)$ & 7 & 3,7 & 10 & 4,6 & 17 & 5,5 \\
\hline \multicolumn{7}{|c|}{ Circunferência muscular do braço $(n=714)$} \\
\hline Desnutrição $(P<5)$ & 26 & 13,9 & 42 & 19,3 & 94 & 22,7 \\
\hline Eutrófico $(P \geq 5)$ & 161 & 86,1 & 176 & 80,7 & 215 & 77,3 \\
\hline \multicolumn{7}{|l|}{ Dobra cutânea tricipital $(n=718)$} \\
\hline Desnutrição $(P<5)$ & 26 & 13,8 & 25 & 11,3 & 21 & 6,8 \\
\hline Eutrófico $(P \geq 5-P \leq 95)$ & 159 & 84,6 & 181 & 81,9 & 272 & 88.0 \\
\hline Obesidade $(P>95)$ & 3 & 1,6 & 15 & 6,8 & 16 & 5,2 \\
\hline
\end{tabular}




\section{Faixa etária}

\begin{tabular}{cccccc}
\hline De 2 & a & 5 anos & De 5 a & 10 anos & De 10 a 19 anos \\
\hline n & $\%$ & n & $\%$ & n & $\%$ \\
\hline
\end{tabular}

Dobra cutânea subescapular $(n=717)$

\author{
Desnutrição $(P<5)$ \\ Eutrófico ( $P \geq 5-P \leq 95)$ \\ Obesidade $(P>95)$
}

Índice de Massa Corpórea $(n=720)$

\begin{tabular}{|c|c|c|c|c|c|c|}
\hline \multicolumn{7}{|l|}{ Indice de Massa Corpórea $(\mathbf{n}=720)$} \\
\hline Magreza acentuada & 4 & 2,1 & - & - & - & - \\
\hline Magreza & 8 & 4,3 & - & - & - & - \\
\hline Eutrofia & 126 & 67,0 & - & - & - & - \\
\hline Risco de sobrepeso & 33 & 17,6 & - & - & - & - \\
\hline Sobrepeso & 11 & 5,9 & - & - & - & - \\
\hline Obesidade & 6 & 3,2 & - & - & - & - \\
\hline Magreza acentuada & - & - & 12 & 5,4 & 20 & 6,4 \\
\hline Magreza & - & - & 12 & 5,4 & 20 & 6,4 \\
\hline Eutrofia & - & - & 142 & 64,3 & 191 & 61,4 \\
\hline Sobrepeso & - & - & 36 & 16,3 & 52 & 16,7 \\
\hline Obesidade & - & - & 15 & 6,8 & 24 & 7,7 \\
\hline Obesidade grave & - & - & 4 & 1,8 & 4 & 1,3 \\
\hline \multicolumn{7}{|l|}{ Estatura para Idade $(n=720)$} \\
\hline Muito baixa estatura para a idade & 8 & 4,3 & - & - & 4 & 1,3 \\
\hline Baixa estatura para a idade & 6 & 3,2 & 7 & 3,2 & 19 & 6,1 \\
\hline Estatura adequada para a idade & 174 & 92,6 & 214 & 96,8 & 288 & 92,6 \\
\hline
\end{tabular}

Fonte: Afonso ${ }^{16}$.

Legenda: ANSGP = Avaliação Nutricional Subjetiva Global Pediátrica.

\section{DISCUSSÃO}

Esses dados transversais representam parte de uma coorte multicêntrica, inédita no país, e mostram que a prevalência em qualquer grau de desnutrição obtida pela ANSGP foi observada entre $25,9 \%$ e $40,1 \%$ das crianças nas diferentes faixas etárias de todas as Regióes do Brasil. Uma diferença expressiva na prevalência de desnutrição foi observada na avaliação nutricional pelo IMC/I, por meio da qual $13 \%$ da amostra nacional foi diagnosticada com desnutrição. Por outro lado, 24,9 \% a 26,7\% dos participantes foram classificados com sobrepeso e obesidade por meio do IMC/I, considerando todas as idades e Regióes.

Os dados da composição corporal, realizada pela CMB, corroboraram a avaliação nutricional subjetiva e apontaram um maior comprometimento da massa magra, de 20\% a $24 \%$ em todas as idades, em relação à massa gorda. Essa desigualdade na prevalência da inadequação do estado nutricional mostra primeiramente a grande divergência que

$\begin{array}{llllll}10 & 5,3 & 15 & 6,8 & 24 & 7,7 \\ 156 & 83,0 & 186 & 85,0 & 269 & 86,8 \\ 22 & 11,7 & 18 & 8,2 & 17 & 5,5\end{array}$




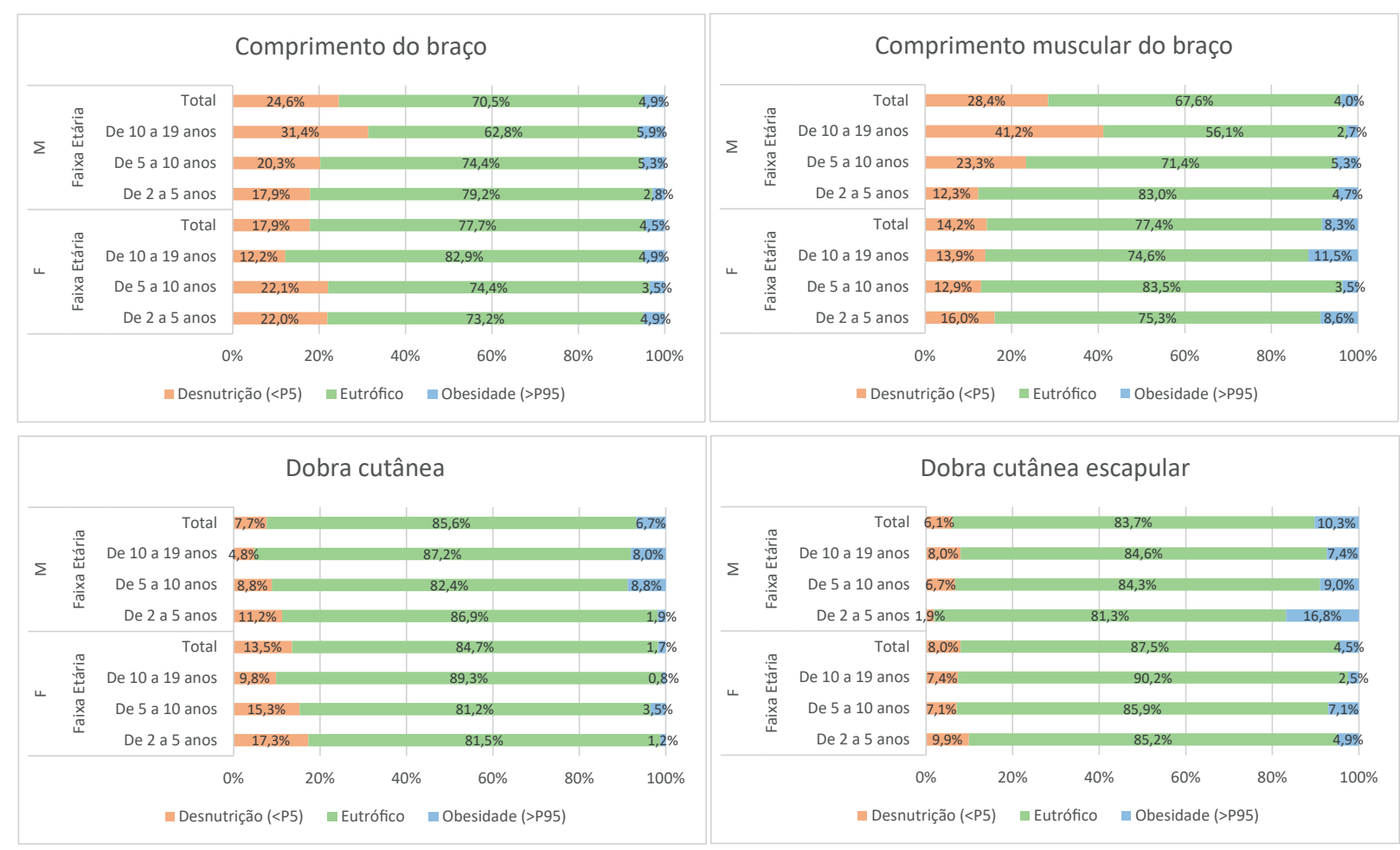

Figura 1. Estado nutricional de acordo com a composição corporal das medidas cutâneas segundo sexo e faixa etária do IBNOPe. Brasil

Fonte: Afonso ${ }^{16}$.

Legendas: $M=$ Masculino; $F=$ Feminino.

Risco elevado de complicaçóes relacionados ao tratamento, redução da tolerância à terapia, dificuldade na cicatrização de feridas, alteraçôes no metabolismo do quimioterápico, maiores chances de infecçôes, desfechos desfavoráveis com menor taxa de sobrevida estão entre os fatores mais citados relacionados ao comprometimento nutricional presente tanto no momento do diagnóstico como ao longo do tratamento do câncer infantojuvenil ${ }^{9,24,27-29}$.

Vale destacar que o déficit nutricional pode inclusive estar presente naqueles pacientes com excesso de peso, e estes tendem a serem subestimados em relação aos riscos da condiçáo nutricional desfavorável e, muitas vezes, ficam à margem de uma intervenção nutricional adequada. Outra atençáo deve ser observada, pois a inadequação nutricional na infância compromete os requisitos fisiológicos necessários para o crescimento e desenvolvimento físico e neurológico e pode favorecer o surgimento ou agravamento de doenças crônicas com impacto negativo na qualidade vida desses indivíduos ${ }^{30}$.

Ainda que os dados mundiais apontem para maior prevalência de sobrepeso e obesidade em crianças, é necessário ponderar que medidas objetivas para avaliaçáo do estado nutricional em crianças hospitalizadas com câncer podem não levar em conta todas as variáveis que uma condição clínica deve considerar para identificar a desnutrição ${ }^{31}$. Em indivíduos saudáveis, a avaliação nutricional por antropometria em crianças é bem reconhecida por acompanhar o crescimento e a saúde da criança e do adolescente, assim como detectar precocemente distúrbios nutricionais, seja desnutrição ou obesidade, sendo considerada essencial para o conhecimento das condiçôes de saúde dos pacientes pediátricos.

Em oncologia pediátrica, segundo Sala et al. ${ }^{13}$, todos os métodos de avaliaçáo nutricional que dependem do peso corporal são problemáticos para uso em crianças, pois, em alguns casos, as massas tumorais podem chegar a $10 \%$ do peso corporal e subestimarem a gravidade da desnutrição. Embora o IMC/I seja amplamente utilizado no diagnóstico nutricional desses pacientes, seu uso como método de avaliação nutricional na prática clínica para pacientes com câncer é bastante desencorajado, pois ele não é capaz de distinguir massa gorda de massa livre de gordura e edemas ${ }^{32}$. E as oscilaçốes no tecido magro e na massa gorda podem afetar o volume de distribuição da quimioterapia no organismo e assim causar modificação na depuração de drogas da circulação sistêmica; por esse motivo, trazem preocupação métodos errôneos de avaliação nutricional ${ }^{33,34}$.

O grande desafio na avaliação nutricional em crianças com câncer ocorre exatamente porque esses pacientes apresentam características que são muito peculiares, como alteraçôes metabólicas, grandes volumes de massas tumorais, edema em virtude das altas doses de corticosteroides por período prolongado, além de efeitos 
colaterais da terapia antineoplásica, principalmente aqueles que afetam o trato gastrointestinal, como mucosites, hiporexia, náuseas, vômitos e diarreia ${ }^{13,35}$. Todos são condiçôes clínicas inerentes tanto à doença como à agressividade do tratamento e são, portanto, comprometedores do estado nutricional, cuja avaliação nutricional objetiva pode falhar.

Nesse contexto, a ANSGP é vista como o instrumento mais adequado por casa da sua capacidade de avaliar crianças em diferentes condiçóes crônicas e sistêmicas, complexidade clínica e uma ampla variedade de comorbidades e doenças subjacentes que envolvem o trato gastrointestinal, uma vez que mostrou, em estudo original, melhores associaçóes com desfechos em pacientes internados ${ }^{31,36}$. Esse instrumento engloba, na sua avaliação, domínios de adequação do peso e estatura; ingestão dietética; frequência e duração dos sintomas gastrintestinais; capacidade funcional; estresse metabólico; e exame físico relacionado à faixa etária pediátrica, mostrando uma capacidade mais abrangente da avaliaçáo nutricional, em especial no paciente pediátrico com câncer ${ }^{17,18,31}$. Entre esses domínios, é importante destacar a relevância do exame físico, focado na nutrição e observação de sinais de perda de estoques de gordura, perda muscular e edema, seguindo um processo lógico e sequencial com uma abordagem da cabeça aos pés, condição imprescindível na avaliação da criança com câncer.

Em relação à avaliação da composição corporal, observou-se um comprometimento de massa muscular esquelética pela antropometria em torno de $20 \%$ a $24 \%$ da amostra em todas as faixas etárias. A perda de massa magra é um elemento básico do fenótipo sarcopênico, podendo resultar na síndrome da fragilidade, pressupondo um envelhecimento prematuro, suscetibilidade a intercorrências clínicas, diminuição da capacidade funcional e alteração da homeostase, como ocorre em adultos com câncer ${ }^{37}$. As alteraçôes na composição corporal podem afetar a absorção do medicamento, diminuir o metabolismo oxidativo, reduzir a taxa de filtração glomerular e, com isso, aumentar as concentraçôes plasmáticas de fármacos e potencialmente a toxicidade ${ }^{32}$. A predição da má nutrição pela medida de composição corporal mediante antropometria do braço tem sido utilizada em crianças com câncer, e sugerida como método adicional de avaliaçáo nutricional, sobretudo em países de baixa e média rendas, pois trata-se de medida simples, praticável, pouco invasiva e economicamente viável $^{32,35,38,39}$.

Um estudo realizado no Brasil de investigação do estado nutricional concluiu que a $\mathrm{CB}$ detectou mais pacientes com desnutriçáo do que o $\mathrm{IMC} / \mathrm{I}^{26}$. Os autores observaram ainda que o IMC/I foi o método que menos identificou a desnutrição em relação aos outros critérios empregados.
Embora seja de conhecimento dos autores a desigualdade de renda existente nas várias Regiôes do país, o presente estudo não abordou dados sobre condiçôes socioeconômicas da amostra, o que pode ser considerado uma limitaçấo no estudo. No entanto, acredita-se que, pelo fato de o estudo ter sido realizado em Centros de Referência para tratamento do câncer infantil no Brasil, que contam com equipe multiprofissional e apoio da rede de voluntariado e casas de apoio que fornecem infraestrutura aos pacientes e família, além dos benefícios concedidos pelo governo, constituídos como direito a todo indivíduo em tratamento de câncer no país sem renda, esse dado náo representa fragilidade à pesquisa, embora pudesse enriquecê-la.

Este é o único estudo multicêntrico e multirregional que determinou a prevalência do estado nutricional na criança e adolescente com câncer no país. Isso é fundamental para contribuição às políticas públicas em âmbito nacional e para que gestores possam estabelecer prioridades na atenção à criança com câncer, além disso permite favorecer a sistematização e gestão da assistência nutricional em oncologia pediátrica.

A avaliação do estado nutricional é indispensável não somente no momento do diagnóstico como ao longo do tratamento e deve ser contínua para garantir o crescimento e o desenvolvimento normal da criança e otimizar os resultados clínicos, não a identificar compromete principalmente as intervençóes nutricionais eficazes na modificação dessa condição nutricional ${ }^{29,40}$. Entretanto, a avaliação nutricional para esses pacientes requer critérios mais ampliados de diagnóstico que levem em consideração a avaliação da composição corporal capaz de identificar as diferenças de tecidos muscular e adiposo e permitir o diagnóstico de sarcopenia na prática clínica.

\section{CONCLUSÃO}

O IBNOPe mostrou que a prevalência de desnutrição nesses pacientes é grande e variada entre as Regióes, e a ANSGP parece identificar melhor a desnutrição, demonstrando que, para esses pacientes, um método adicional, além da antropometria, pode ser mais adequado para o diagnóstico nutricional. Sendo assim, faz-se necessária a utilização de métodos com maior abrangência de capacidade diagnóstica e que leve em consideração as especificidades da criança com câncer.

\section{CONTRIBUIÇÕES}

Nivaldo Barroso de Pinho, Wanélia Vieira Afonso, Patricia de Carvalho Padilha, Wilza Arantes Ferreira Peres, Arthur Orlando Corrêa Schilithz, Renata Brum Martucci 
e Viviane Dias Rodrigues contribuíram substancialmente na concepção e planejamento do estudo; na obtenção, análise e interpretação dos dados; assim como na redação e revisão crítica. Carolina Fernandes de Macedo Soares e Juliana Silva do Nascimento Braga contribuíram na obtenção, coleta e revisão dos dados. Todos os autores aprovaram a versão final a ser publicada.

\section{DECLARAÇÃO DE CONFLITO DE INTERESSES}

Nada a declarar.

\section{FONTES DE FINANCIAMENTO}

Apoio financeiro da Sociedade Brasileira de Nutrição Oncológica (SBNO) para treinamento e confecção da plataforma on-line disponibilizada no site da SBNO, além do apoio dos Centros de Referência das Instituiçóes participantes com disponibilidade de materiais da rotina hospitalar.

\section{AGRADECIMENTOS}

Aos pacientes e seus familiares por colaborarem com a ciência e com este estudo. Aos nutricionistas que participaram da coleta de dados: Rafaelle de Barros Caxiano Chissini, Fernanda Bassan Lopes da Silva, Roseli Bottura, Mirelle Sifroni Farias, Clara Pereira Murta de Almeida, Francine Cristina Cordeiro, Lorena de Oliveira Goulart Barsand, Kellen Benites Nemetz, Laura de Carvalho Bastos Domingues, Luciane Beitler da Cruz, Daniela Mayumi Kawano Azuma, Fabiana Rodrigues Pires, Marcia Vanessa Viola Reis, Aline Maria Luna, Maria do Socorro Lira Paes Batista, Rita de Cássia Bahia Viana, Isadora Cordeiro dos Prazeres, Dayse Márcia de Sousa Gurjão, Amanda Munay de Andrade Pimentel, Isa Leandro Soares Aquino Silva, Maria Amélia Marques Dantas, Giovana Lourençáo, Lenira Maximiano Hoff, Rafaela Mazzo, Mayana Costa Ponce, Josilene Venâncio da Silva, Silvia Patrícia de Oliveira Silva Bacalhau, Mary Emilly Vitória da Rocha Veloso, Iago Alves Miranda Santos, Daieni Fernandes, Dafne Pavão Schattschneider e Jessica Lesina de Abreu.

\section{REFERÊNCIAS}

1. Ward ZJ, Yeh JM, Bhakta N, et al. Estimating the total incidence of global childhood cancer: a simulation-based analysis. Lancet Oncol. 2019;20(4):483-93. doi: https:// doi.org/10.1016/S1470-2045(18)30909-4

2. Rodriguez-Galindo C, Friedrich P, Alcasabas P, et al. Toward the cure of all children with cancer through collaborative efforts: pediatric oncology as a global challenge. J Clin Oncol. 2015;33(27):3065-73. doi: https://doi.org/10.1200/JCO.2014.60.6376

3. Centro de Informação das Naçôes Unidas para o Brasil [Internet]. Rio de Janeiro: UNIC; c2020. OMS: um em cada três países de baixa e média renda enfrenta extremos da má nutriçáo; 2019 dez 18 [acesso 2020 set 8]. Disponível em: https://nacoesunidas.org/oms-umem-cada-tres-paises-de-baixa-e-media-renda-enfrentaextremos-da-ma-nutricao

4. Instituto Nacional de Câncer José Alencar Gomes da Silva [Internet]. Rio de Janeiro: INCA; [data desconhecida]. Tipos de Câncer: câncer infantojuvenil; [modificado 2021 mar 4; acesso 2020 set 8]. Disponível em: https:// www.inca.gov.br/tipos-de-cancer/cancer-infantojuvenil

5. Instituto Brasileiro de Geografia e Estatística [Internet]. Rio de janeiro: IBGE; [data desconhecida]. Pesquisa de orçamentos familiares 2008-2009. Análise do consumo alimentar no Brasil; 2009 [acesso 2020 set 7]. Disponível em: https://www.ibge.gov.br/estatisticas/sociais/ rendimento-despesa-e-consumo/9050-pesquisa-deorcamentos-familiares.html?edicao $=9058 \& \mathrm{t}=$ downloads

6. Bloch KV, Klein CH, Szklo M, et al. ERICA: prevalências de hipertensão arterial e obesidade em adolescentes brasileiros. Rev Saúde Pública. 2016;50(Suppl 1):9s. doi: https://doi.org/10.1590/S01518-8787.2016050006685

7. Ministério da Saúde (BR), Secretaria de Atenção à Saúde, Departamento de Atenção Básica. Política Nacional de alimentação e nutrição. Brasília, DF: Ministério da Saúde; 2013.

8. World Health Organization. Reducing stunting in children: equity considerations for achieving the Global Nutrition Targets 2025 [Internet]. Geneva: WHO; 2018 [cited 2020 Sept 10]. Available from: https://www.who. int/publications/i/item/9789241513647

9. Co-Reyes E, Li R, Huh W, et al. Malnutrition and obesity in pediatric oncology patients: causes, consequences, and interventions. Pediatr Blood Cancer. 2012;59(7):1160-7. doi: https://doi.org/10.1002/pbc.24272

10. Brinksma A, Roodbol PF, Sulkers E, et al. Changes in nutritional status in childhood câncer patients: a prospective cohort study. Clin Nutr. 2015;34(1):66-73. doi: https://doi.org/10.1016/j.clnu.2014.01.013

11. Srivastava R, Pushpam D, Dhawan D, et al. Indicators of malnutrition in children with cancer: a study of 690 patients from a tertiary care cancer center. Indian J Cancer. 2015;52(2):199-201. doi: https://doi. org/10.4103/0019-509X.175825

12. Viani K, Albuquerque L, Barr RD, et al. Nutrition of children with cancer in Brazil: a systematic review. JCO Glob Oncol. 2020;6:242-59. doi: https://doi. org/10.1200/JGO.19.00285

13. Sala A, Rossi E, Antillon F, et al. Nutritional status at diagnosis is related to clinical outcomes in children and adolescents with cancer: a perspective from Central 
America. Eur J Cancer. 2012;48(2):243-52. doi: https:// doi.org/10.1016/j.ejca.2011.06.006

14. Instituto Nacional de Câncer José Alencar Gomes da Silva. Inquérito brasileiro de nutrição oncológica [Internet]. Rio de Janeiro: INCA; 2013 [acesso 2020 set 8]. Disponível em: https://www.inca.gov.br/sites/ufu.sti. inca.local/files//media/document//inquerito-brasileironutricao-oncologica.pdf

15. Instituto Nacional de Câncer José Alencar Gomes da Silva. Inquérito luso-brasileiro de nutrição oncológica do idoso: um estudo multicêntrico [Internet]. Rio de Janeiro: INCA; 2015 [acesso 2020 set 8]. Disponível em: https://www.inca.gov.br/sites/ufu.sti.inca.local/files// media/document//inquerito-lusobrasileiro-de-nutricaooncologica-completo.pdf

16. Afonso WV. Inquérito brasileiro de nutrição oncológica em pediatria: um estudo multicêntrico de base hospitalar [tese na Internet]. Rio de Janeiro, RJ: Instituto de Nutrição Josué de Castro, Universidade Federal do Rio de Janeiro; 2020 [acesso $2020 \mathrm{dez}$ 19]. 178 p. Disponível em: chrome-extension://efaidnbmnnnibpcajpcglclefindmkaj/ viewer.html?pdfurl=http\%3 A \% 2F\% 2 Fwww. ppgn.ufrj.br\%2Fwp-content $\% 2$ Fuploads $\%$ 2F2021\%2F04\%2FWanelia-Vieira-Afonso-tese. pdf\&clen $=3161400 \&$ chunk=true

17. Saraiva DCA, Afonso WV, Pinho NB, et al. Equivalência semântica do Questionário Pediatric Subjective Global Nutritional Assessment para triagem nutricional em pacientes pediátricos com câncer. Rev Nutr. 2016;29(2):211-27. doi: https://doi.org/10.1590/167898652016000200006

18. Saraiva DCA, Afonso WV, Pinho NB, et al. Cross-cultural adaptation and content validation into Portuguese of the Subjective Global Nutritional Assessment for pediatric patients hospitalized with cancer. Rev Nutr. 2017;30(3):307-20. doi: https://doi.org/10.1590/167898652017000300004

19. Ministério da Saúde (BR), Secretaria de Atenção à Saúde, Departamento de Atenção Básica. Orientações para a coleta e análise de dados antropométricos em serviços de saúde: norma técnica do Sistema de Vigilância Alimentar e Nutricional - SISVAN. Brasília, DF: Ministério da Saúde; 2011. (Série G. Estatística e Informação em Saúde).

20. World Health Organization. WHO child growth standards: length/height-for-age, weight-for-age, weightfor-length, weight-for-height and body mass index-forage: methods and development. Geneva: WHO Press; 2006.

21. WHO Anthro Survey Analyser [Internet]. Version 3.2.2. Geneva: WHO. c2011 - [cited 2018 Mar 13]. Available from: doi: http://www.who.int/childgrowth/software/en

22. Frisancho AR. Anthropometric standards for the assessment of growth and nutritional status. Ann Arbor, MI: University of Michigan Press; 1990.
23. Osterkamp LK. Current perspective on assessment of human body proportions of relevance to amputees. J Am Diet Assoc. 1995;95(2):215-8. doi: https://doi. org/10.1016/S0002-8223(95)00050-X

24. Joffe L, Dwyer S, Bender JLG, et al. Nutritional status and clinical outcomes in pediatric patients with solid tumors: a systematic review of the literature. Semin Oncol. 2019;46(1):48-56. doi: https://doi. org/10.1053/j.seminoncol.2018.11.005

25. Lemos PSM, Oliveira FLC, Caran EMM. Nutritional status of children and adolescents at diagnosis of hematological and solid malignancies. Rev Bras Hematol Hemoter. 2014;36(6):420-3. doi: https://doi. org/10.1016/j.bjhh.2014.06.001

26. Viani K, Barr RD, Odone Filho V, et al. Nutritional status at diagnosis among children with cancer referred to a nutritional service in Brazil. Hematol Transfus Cell Ther. 2021;43(4):389-95. doi: https://doi.org/10.1016/j. htct.2020.04.008

27. Hingorani P, Seidel K, Krailo M, et al. Body Mass Index (BMI) at diagnosis is associated with surgical wound complications in patients with localized osteosarcoma: a report from the Children's Oncology Group. Pediatr Blood Cancer. 2011;57(6):939-42. doi: https://doi. org/10.1002/pbc.23129

28. Loeffen EAH, Brinksma A, Miedema KGE, et al. Clinical implications of malnutrition in childhood cancer patients -- infections and mortality. Support Care Cancer. 2015;23(1):143-50. doi: https://doi.org/10.1007/ s00520-014-2350-9

29. Orgel E, Sposto R, Malvar J, et al. Impact on survival and toxicity by duration of weight extremes during treatment for pediatric acute lymphoblastic leukemia: a report from the Children's Oncology Group. J Clin Oncol. 2014;32(13):1331-7. doi: https://doi.org/10.1200/ JCO.2013.52.6962

30. Diller L, Chow EJ, Gurney JG, et al. Chronic disease in the childhood cancer survivor study cohort: a review of published findings. J Clin Oncol. 2009;27(14):2339-55. doi: https://doi.org/10.1200/JCO.2008.21.1953

31. Secker DJ, Jeejeebhoy KN. How to perform subjective global nutritional assessment in children. J Acad Nutr Diet. 2012;112(3):424-31.e6. doi: https://doi. org/10.1016/j.jada.2011.08.039

32. Murphy-Alford AJ, Prasad M, Slone J, et al. Perspective: creating the evidence base for nutritional support in childhood cancer in low- and middle-income countries: priorities for body composition research. Adv Nutr. 2020;11(2):216-23. doi: https://doi.org/10.1093/ advances/nmz095

33. Yip C, Dinkel C, Mahajan A, et al. Imaging body composition in cancer patients: visceral obesity, sarcopenia and sarcopenic obesity may impact on clinical outcome. Insights Imaging. 2015;6(4):489-97. doi: https://doi.org/10.1007/s13244-015-0414-0 
34. Behan JW, Avramis VI, Yun JP, et al. Diet-induced obesity alters vincristine pharmacokinetics in blood and tissues of mice. Pharmacol Res. 2010;61(5):385-90. doi: https:// doi.org/10.1016/j.phrs.2010.01.007

35. Barr R, Collins L, Nayiager T, et al. Nutritional status at diagnosis in children with cancer. 2. An assessment by arm anthropometry. J Pediatr Hematol Oncol. 2011;33(3):e101-4. doi: https://doi.org/10.1097/ MPH.0b013e3182002a65

36. Secker DJ, Jeejeebhoy KN. Subjective global nutritional assessment for children. Am J Clin Nutr. 2007;85(4):10839. doi: https://doi.org/10.1093/ajcn/85.4.1083

37. Barr RD, Ladas EJ. The role of nutrition in pediatric oncology. Expert Rev Anticancer Ther. 2020;20(2):10916. doi: https://doi.org/10.1080/14737140.2020.171 9834

38. Ladas EJ, Arora B, Howard SC, et al. A framework for adapted nutritional therapy for children with cancer in low- and middle-income countries: a report from the SIOP PODC nutrition working group. Pediatr Blood Cancer. 2016;63(8):1339-48. doi: https://doi. org/10.1002/pbc.26016

39. Bliss J, Lelijveld N, Briend A, et al. Use of mid-upper arm circumference by novel community platforms to detect, diagnose, and treat severe acute malnutrition in children: a systematic review. Glob Health Sci Pract. 2018;6(3):552-64. doi: https://doi.org/10.9745/ GHSP-D-18-00105

40. Joffe L, Ladas EJ. Nutrition during childhood cancer treatment: current understanding and a path for future research. Lancet Child Adolesc Health. 2020;4(6):465-75. doi: https://doi.org/10.1016/S2352-4642(19)30407-9 